\title{
New Inequalities between Information Measures of Network Information Content
}

\author{
Pantelimon-George Popescu, Florin Pop, Alexandru Herişanu, and Nicolae Ţăpuş \\ Computer Science Department, Faculty of Automatic Control and Computers, University Politehnica of Bucharest, \\ Splaiul Independentei 313, Bucharest 060042, Romania \\ Correspondence should be addressed to Florin Pop; florin.pop@cs.pub.ro
}

Received 12 December 2012; Accepted 28 January 2013

Academic Editor: Carlo Cattani

Copyright (c) 2013 Pantelimon-George Popescu et al. This is an open access article distributed under the Creative Commons Attribution License, which permits unrestricted use, distribution, and reproduction in any medium, provided the original work is properly cited.

\begin{abstract}
We refine a classical logarithmic inequality using a discrete case of Bernoulli inequality, and then we refine furthermore two information inequalities between information measures for graphs, based on information functionals, presented by Dehmer and Mowshowitz in (2010) as Theorems 4.7 and 4.8. The inequalities refer to entropy-based measures of network information content and have a great impact for information processing in complex networks (a subarea of research in modeling of complex systems).
\end{abstract}

\section{Introduction}

We first present the classical Shannon's entropy; see [1,2]. Let $X$ be a discrete random variable taking values in $A$, and let $p\left(x_{i}\right)=\operatorname{Pr}\left(X=x_{i}\right)$ be the probability mass function of $X$; then, the Shannon entropy of $X$ is defined by

$$
H(X):=-\sum_{x_{i} \in A} p\left(x_{i}\right) \log \left(p\left(x_{i}\right)\right) \text {. }
$$

We continue with some basic theoretical information about graphs; see $[3,4]$. We consider $G=(V, E),|V|<\infty$ to be a finite undirected graph, where $E \subseteq\left(\begin{array}{c}V \\ 2\end{array}\right)$. $G$ is connected if for any arbitrary vertices $v_{i}, v_{j}$ exists an undirected path from $v_{i}$ to $v_{j}$. Furthermore, we will consider $\mathscr{G}_{U C}$ to be the set of finite undirected and connected graphs. This is usually used in strategies for improving transport efficiency in Network Information Content, including designing efficient routing strategies and making appropriate adjustments to the underlying network structure [5].

Now, taking into account the work presented in $[6,7]$, we define the concept of information functional. Let $G=(V, E) \in$ $\mathscr{G}_{U C}$, and let $S$ initially be an abstract set; then, $f: S \rightarrow R^{+}$ is called the information functional of $G$ (we assume that $f$ is monotonous). The abstract set $S$ can be, for example, the vertex sets, a set of paths, certain subgraphs, and so forth. The set $S$ is used to define the functional $f$ that captures the structural information of graph $G$ and has to be defined concretely.

Using different information functionals to measure the entropy of a graph, we obtain different probability distributions; so, the resulting graph entropies are also different. Further, we define, as in $[6,7]$, the entropy of a graph $G=$ $(V, E) \in \mathscr{G}_{U C}$ with arbitrary vertex labels $v_{i}$, using an arbitrary information functional $f$, to be

$$
I^{f}(G):=-\sum_{i=1}^{|V|} \frac{f\left(v_{i}\right)}{\sum_{j=1}^{|V|} f\left(v_{j}\right)} \log \left(\frac{f\left(v_{i}\right)}{\sum_{j=1}^{|V|} f\left(v_{j}\right)}\right),
$$

and considering a vertex $v_{i} \in V, p^{f}\left(v_{i}\right)=f\left(v_{i}\right) / \sum_{j=1}^{|V|} f\left(v_{j}\right)$ interpreted as vertex probabilities $\left(\sum_{i=1}^{|V|} p^{f}\left(v_{i}\right)=1\right)$, we conclude that

$$
I^{f}(G)=-\sum_{i=1}^{|V|} p^{f}\left(v_{i}\right) \log \left(p^{f}\left(v_{i}\right)\right) .
$$

\section{Refinement of a Classical Logarithmic Inequality}

It is well known from the literature that

$$
\log _{b}(x) \leq \frac{1}{\ln (b)}(x-1), \quad \forall x>0 .
$$


Trying to refine this inequality, we consider the classical discrete Bernoulli inequality, as follows.

Theorem 1 (Bernoulli inequality). Let $a_{i} \geq 0, x_{i}>-1, i=$ $1, \ldots, n$ and $\sum_{i=1}^{n} a_{i} \leq 1$. Then,

$$
\prod_{i=1}^{n}\left(1+x_{i}\right)^{a_{i}} \leq 1+\sum_{i=1}^{n} a_{i} x_{i}
$$

if $a_{i} \geq 1$ or $a_{i} \leq 0$, and if $x_{i}>0$, or $-1<x_{i}<0, i=1, \ldots, n$, then

$$
\prod_{i=1}^{n}\left(1+x_{i}\right)^{a_{i}} \geq 1+\sum_{i=1}^{n} a_{i} x_{i}
$$

Now, using a factorization of a real number, one concludes as follows.

Theorem 2. Let $x>0$ be a real number; then,

$$
\log _{b}(x) \leq \frac{1}{\ln (b)} \sum_{i=1}^{n} x_{i} \leq \frac{1}{\ln (b)}(x-1)
$$

where $n \geq 2$ and $x=\prod_{i=1}^{n}\left(x_{i}+1\right)$, with $x_{i}>0$ if $x>1, x_{i}=0$ if $x=1$, and $0>x_{i}>-1$ if $1>x>0, \forall 1 \leq i \leq n$.

Proof. The inequality becomes an identity for $x=1$; that is, $x_{i}=0, \forall 1 \leq i \leq n$. We will treat the other two cases, when $x>1$ and when $1>x>0$ in the same manner, as follows:

$$
\log _{b}(x)=\log _{b} \prod_{i=1}^{n}\left(x_{i}+1\right)=\sum_{i=1}^{n} \log _{b}\left(x_{i}+1\right)
$$

for which applying the classical logarithmic inequality for $x_{i}+$ $1>0, \forall 1 \leq i \leq n$ yields

$$
\log _{b}(x) \leq \frac{1}{\ln (b)} \sum_{i=1}^{n} x_{i}
$$

and making use of the second part of the discrete Bernoulli inequality, previously presented, we obtain

$$
\frac{1}{\ln (b)} \sum_{i=1}^{n} x_{i} \leq \frac{1}{\ln (b)}\left[\prod_{i=1}^{n}\left(x_{i}+1\right)-1\right]=\frac{1}{\ln (b)}(x-1) \text {, }
$$

and we are done.

\section{Refinement of Dehmer and Mowshowitz Inequalities between Information Measures}

In the work of Dehmer and Mowshowitz [6], by Theorems 4.7 and 4.8 are presented two information inequalities derived assuming only the characteristic properties of the functions involved. Here, we refine those results by using the previous theorem, as follows.
Theorem 3. Let $f$ and $f^{*}$ be information functionals. Then (all logarithms are in base 2),

$$
\begin{aligned}
& I^{f}(G) \geq-\sum_{k=1}^{|V|} p^{f}\left(v_{k}\right) \log \left(p^{f^{*}}\left(v_{k}\right)\right) \\
&-\frac{1}{\ln (2)} \sum_{i=1}^{n} p_{i}^{k} \sum_{k=1}^{|V|} p^{f}\left(v_{k}\right) \\
& \geq-\sum_{k=1}^{|V|} p^{f}\left(v_{k}\right) \log \left(p^{f^{*}}\left(v_{k}\right)\right) \\
&-\frac{1}{\ln (2)} \sum_{k=1}^{|V|} \frac{\left(p^{f}\left(v_{k}\right)\right)^{2}-p^{f}\left(v_{k}\right) p^{f^{*}}\left(v_{k}\right)}{p^{f^{*}}\left(v_{k}\right)} \\
& I^{f^{*}}(G) \leq \frac{1}{\ln (2)} \sum_{i=1}^{n} p_{i}^{k} \sum_{k=1}^{|V|} p^{f^{*}}\left(v_{k}\right) \\
&-\sum_{k=1}^{|V|} p^{f^{*}}\left(v_{k}\right) \log \left(p^{f}\left(v_{k}\right)\right) \\
& \leq \frac{1}{\ln (2)} \sum_{k=1}^{|V|}\left[p^{f}\left(v_{k}\right)-p^{f^{*}}\left(v_{k}\right)\right. \\
&\left.-\ln (2) p^{f^{*}}\left(v_{k}\right) \log \left(p^{f}\left(v_{k}\right)\right)\right],
\end{aligned}
$$

where $n \geq 2$ and $p^{f}\left(v_{k}\right) / p^{f^{*}}\left(v_{k}\right)=\prod_{i=1}^{n}\left(p_{i}^{k}+1\right), \forall 1 \leq k \leq$ $|V|$, with $\forall 1 \leq k \leq|V|, p_{i}^{k}>0$ if $p^{f}\left(v_{k}\right)>p^{f^{*}}\left(v_{k}\right), p_{i}^{k}=0$ if $p^{f}\left(v_{k}\right)=p^{f^{*}}\left(v_{k}\right)$, and $0>p_{i}^{k}>-1$ if $p^{f}\left(v_{k}\right)<p^{f^{*}}\left(v_{k}\right)$, $\forall 1 \leq i \leq n$.

Proof. Applying Theorem 2 for $x=p^{f}\left(v_{k}\right) / p^{f^{*}}\left(v_{k}\right)>0$ yields that

$$
\begin{aligned}
& \log \left(p^{f}\left(v_{k}\right)\right)-\log \left(p^{f^{*}}\left(v_{k}\right)\right) \\
& \quad \leq \frac{1}{\ln (2)} \sum_{i=1}^{n} p_{i}^{k} \\
& \quad \leq \frac{1}{\ln (2)} \frac{p^{f}\left(v_{k}\right)-p^{f^{*}}\left(v_{k}\right)}{p^{f^{*}}\left(v_{k}\right)}, \quad \forall 1 \leq k \leq|V| .
\end{aligned}
$$

By multiplying this inequality first with $-p^{f}\left(v_{k}\right)$ and then with $p^{f^{*}}\left(v_{k}\right)$, we obtain

$$
\begin{aligned}
-p^{f}\left(v_{k}\right) & \log \left(p^{f}\left(v_{k}\right)\right) \\
\geq & -\frac{1}{\ln (2)} \sum_{i=1}^{n} p_{i}^{k} \cdot p^{f}\left(v_{k}\right) \\
& -p^{f}\left(v_{k}\right) \log \left(p^{f^{*}}\left(v_{k}\right)\right)
\end{aligned}
$$




$$
\begin{gathered}
\geq-\frac{1}{\ln (2)} \frac{\left(p^{f}\left(v_{k}\right)\right)^{2}-p^{f}\left(v_{k}\right) p^{f^{*}}\left(v_{k}\right)}{p^{f^{*}\left(v_{k}\right)}} \\
-p^{f}\left(v_{k}\right) \log \left(p^{f^{*}}\left(v_{k}\right)\right), \\
-p^{f^{*}}\left(v_{k}\right) \log \left(p^{f^{*}}\left(v_{k}\right)\right) \\
\leq \frac{1}{\ln (2)} \sum_{i=1}^{n} p_{i}^{k} \cdot p^{f^{*}}\left(v_{k}\right) \\
-p^{f^{*}}\left(v_{k}\right) \log \left(p^{f}\left(v_{k}\right)\right) \\
\leq \frac{1}{\ln (2)}\left(p^{f}\left(v_{k}\right)-p^{f^{*}}\left(v_{k}\right)\right) \\
\quad-p^{f^{*}}\left(v_{k}\right) \log \left(p^{f}\left(v_{k}\right)\right), \\
\forall 1 \leq k \leq|V| .
\end{gathered}
$$

[6] M. Dehmer and A. Mowshowitz, "Inequalities for entropybased measures of network information content," Applied Mathematics and Computation, vol. 215, no. 12, pp. 4263-4271, 2010.

[7] M. Dehmer, "Information processing in complex networks: graph entropy and information functionals," Applied Mathematics and Computation, vol. 201, no. 1-2, pp. 82-94, 2008.

Finally, summating after $k$ yields the wanted results.

\section{Conclusions}

As a main result of this paper, we improved two inequalities between entropy-based measures, this giving us a way of bounding measures of network properties. A good example for this purpose is presented into [6], where, using concrete information functionals, the corresponding entropies for the graphs in a set of 2265 nonisomorphic chemical graphs, that is, in MS2265, are computed. Here, new stronger lower and, respectively, upper bounds for entropy-based measures are given and thus, by knowing the limitations of such measures, we narrow down the set of expected values. The utility of inequalities between measures of structural information content is found in problem solving through problem transformation, as is also spotted in [6].

\section{Acknowledgment}

This work was supported and founded by the project "ERRICEmpowering Romanian Research on Intelligent Information Technologies/FP7-REGPOT-2010-1", ID: 264207.

\section{References}

[1] R. Ash, Information Theory, Interscience, New York, NY, USA, 1965.

[2] T. M. Cover and J. A. Thomas, Elements of Information Theory, John Wiley \& Sons, 2006.

[3] F. Buckley and F. Harary, Distance in Graphs, Addison-Wesley, 1990.

[4] F. Harary, Graph Theory, Addison-Wesley, Reading, Mass, USA, 1969.

[5] S. Chen, W. Huang, C. Cattani, and G. Altieri, "Traffic dynamics on complex networks: a survey," Mathematical Problems in Engineering, vol. 2012, Article ID 732698, 23 pages, 2012. 


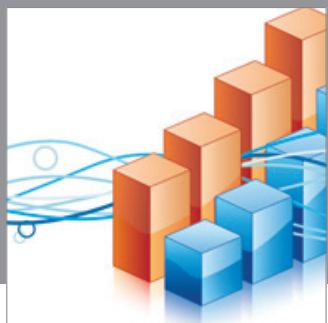

Advances in

Operations Research

mansans

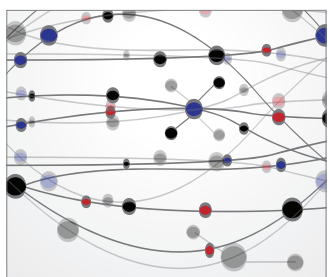

The Scientific World Journal
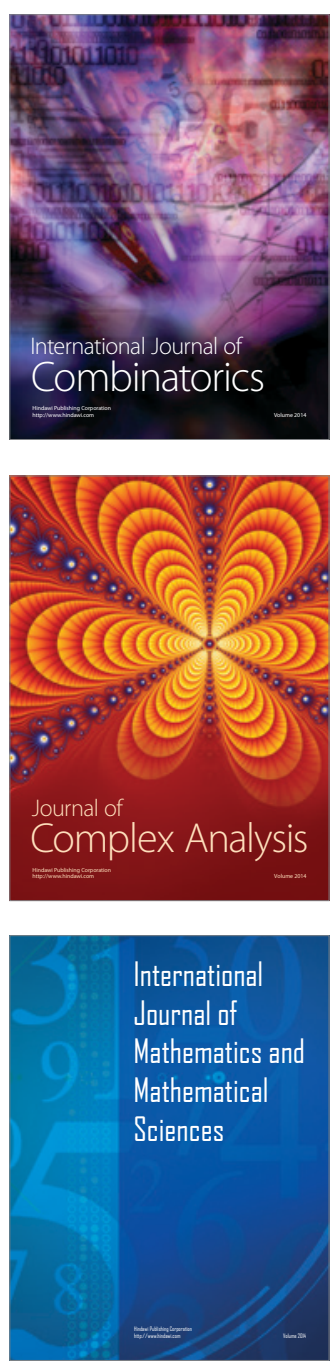
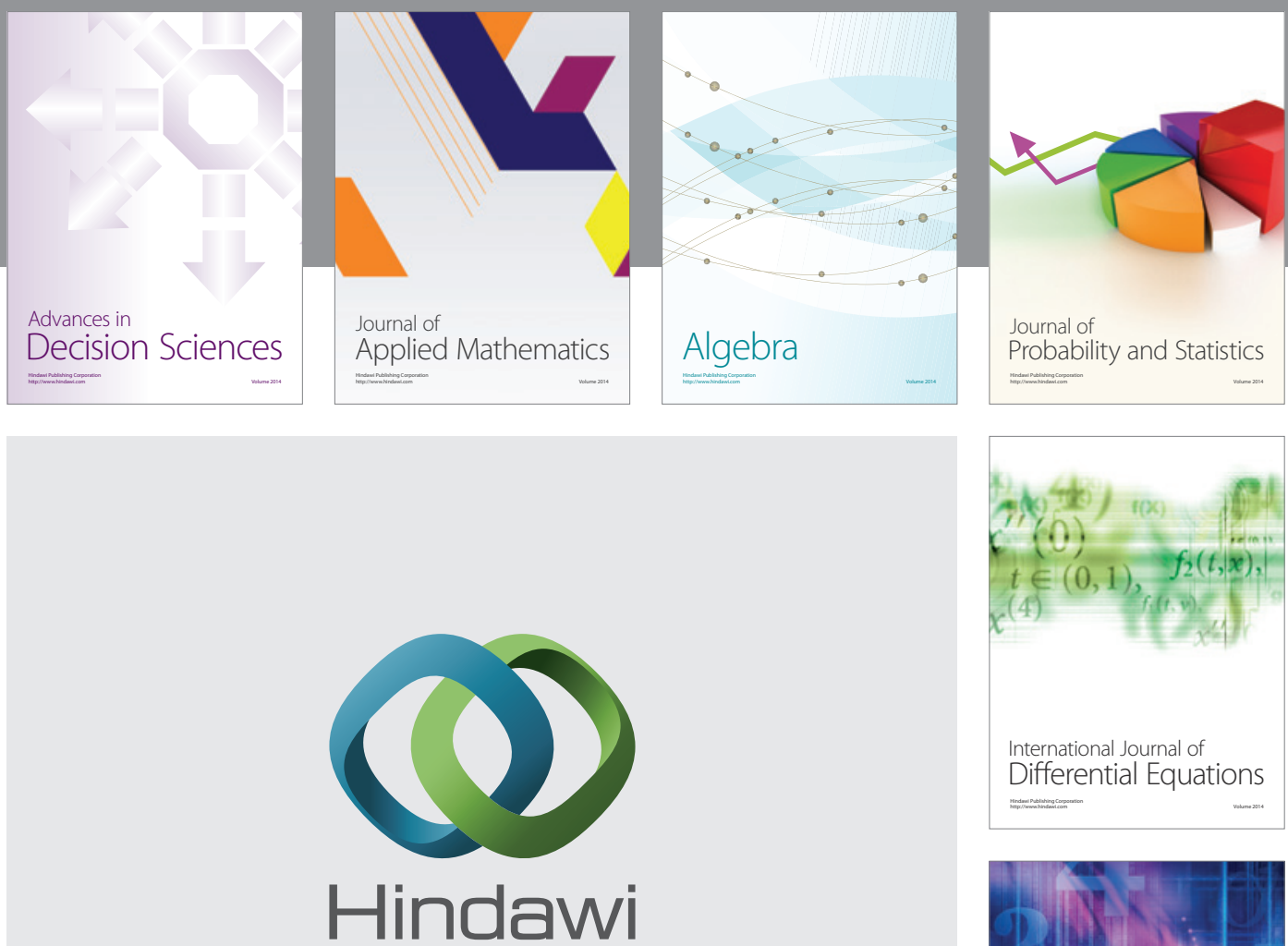

Submit your manuscripts at http://www.hindawi.com
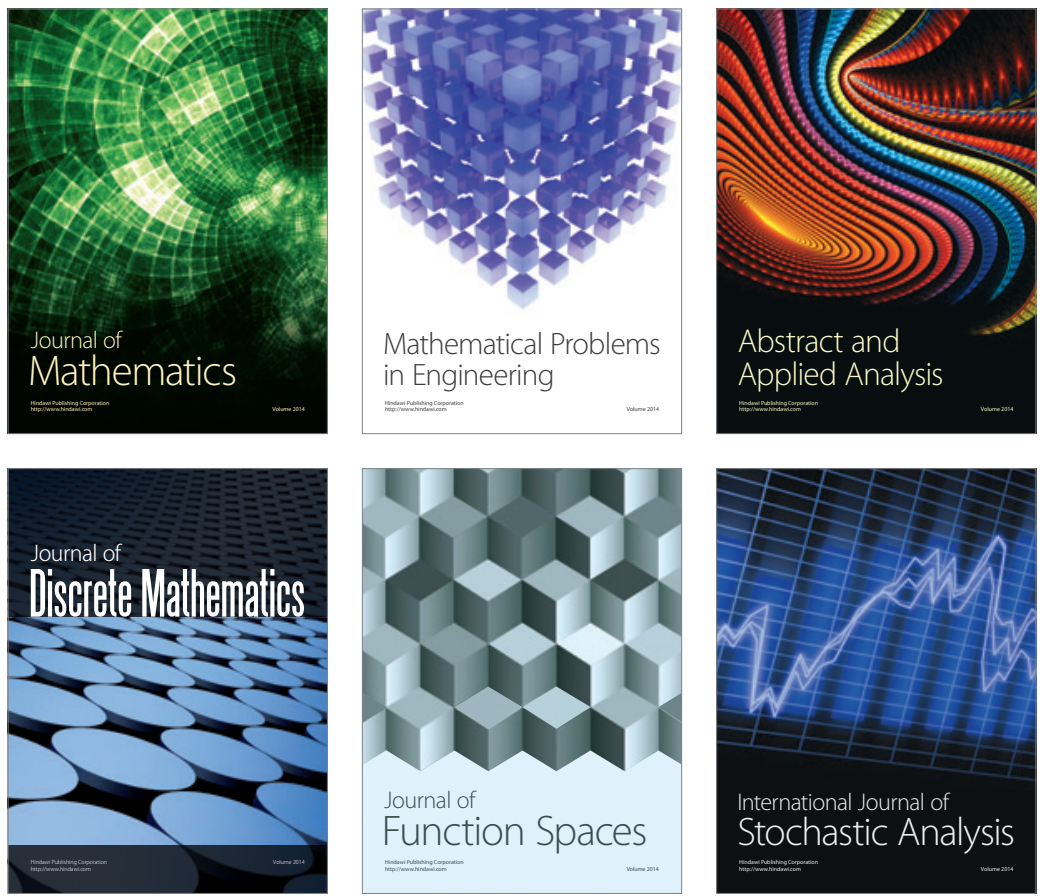

Journal of

Function Spaces

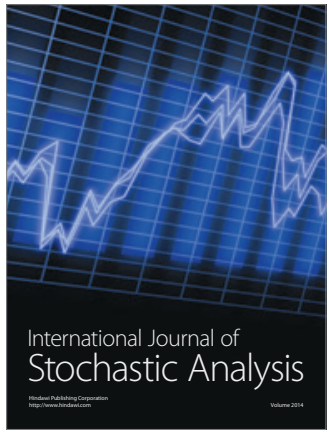

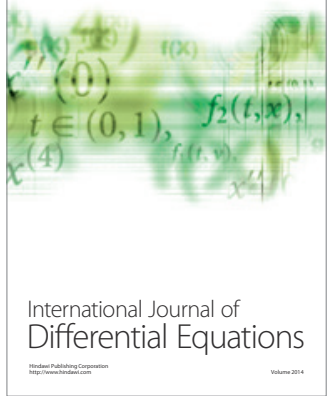
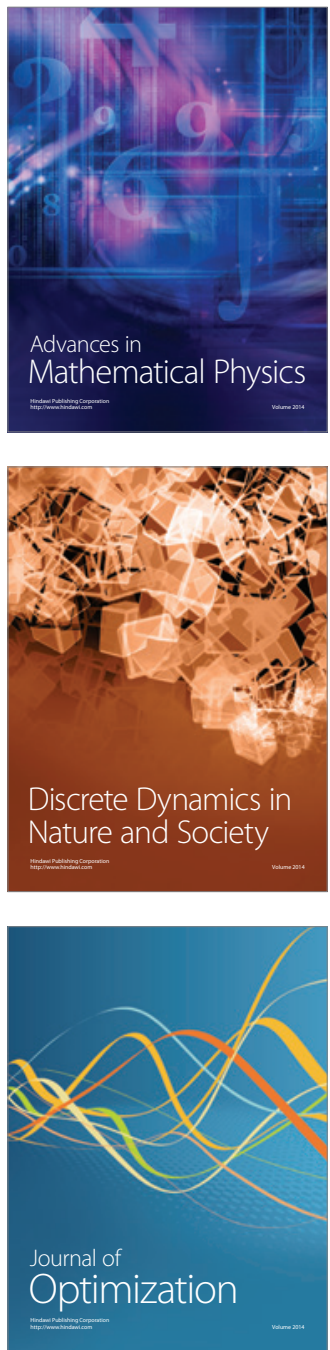